\title{
Fullerite-Diamond Transition Under Shock Loading
}

\author{
A.A. Dityat'ev, O.G. Epanchintsev, A.E. Korneev and V.F. Nesterenko
}

Moscow State University, 119899, Moscow, Russia

\section{INTRODUCTION}

In 1985 Kroto and Smalley and their research group synthesized stable carbon clusters - fullerenes, consisting of 60 carbon atoms /1/. The recent discoveries that bulk quantities of the fullerene molecules can be crystallized $/ 2 /$ have promoted investigations into the physical properties of both $\mathrm{C} 60$ molecules and $\mathrm{C} 60$ crystals (fullerites). C60 molecules have a highly symmetrical pseudospherical structure, which is extremely stable, withstanding hydrostatic pressures of up to at least $20 \mathrm{GPa} / 3 /$. The nature of phase transitions in fullerite is of great interest over a wide range of pressures and temperatures, because of the unusual structure of the fullerenes. New high-pressure carbon phases have recently been observed experimentally or predicted theoretically, including n-diamond, transparent pressured graphite $/ 4 /$. On the application of high shock pressures, the fullerites might transform into as yet undiscovered phases. Since 1991 several publications have appeared on experimental investigations of phase transformations in fullerites at high pressures, including high shock pressures /3-7/. The formation of different phases was detected, in particular diamond-like carbon phases $15-7$, which is of peculiar interest because of the theoretical prediction that fullerite subjected to high-pressure loading can form a solid harder than diamond /8/. The most interesting problem among those mentioned above is the fulleriteto-diamond transformation, which may be realized using explosive systems. The beneficial feature of highenergy explosion treatment of solids is the very high concentration of defects generated in the crystal lattice and the high level of internal stress, which promotes phase transformations and substantially affects the evolution of the material structure.

During the last two years, several publications on the fullerite-to-diamond phase transition at high shock pressure have appeared $15-7 /$. In $/ 6 /$, a thin film of fullerite, $\mathrm{C60}$, was subjected to shock-wave loading in a gas gun up to $69 \mathrm{GPa}$. TEM investigations identified a small amount of cubic diamond and fine-grained ndiamond (5-35 nm). The period of loading was probably quite sufficient for decomposition of $\mathrm{C} 60$ but too short for diamond growth.

The mixture of $\mathrm{C} 60$ and copper powder was shockcompressed in the range of $12-37 \mathrm{GPa} / 71$. Initial fullerene powder contains a small amount of $\mathrm{C70}$. Fullerenes were dispersed in a copper matrix as an efficient quenching medium. Compacts were studied using X-ray structure analysis, TEM and Raman spectroscopy. Pressure-loaded samples consisted of C60 and graphite at 12-19 $\mathrm{GPa}$ and of graphite and diamond (5-25 nm) at 20-37 GPa $7 /$. The authors propose that the finest diamond particles, formed from fullerenes at high pressure, can never be retained at high speed quenching, and they transform into graphite.

It has been proposed that the fullerite-to-diamond transition be stimulated by nonhydrostatic loading, e.g., by shear strain 15/: compression under nonhydrostatic conditions at $20 \mathrm{GPa}$ stimulated instantaneous synthesis of diamond powder (2-100 nm) from C60.

The long-term objectives of this work are investigations of phase transformations in fullerites on the application of high shock pressure and realization of a new method of diamond synthesis. The gas gun method of shock-wave treatment used in $/ 4,6,7 /$ is very 
complicated to carry out in practice.

We chose a method based on a condensed explosive, because it is very promising for the industrial technology. The fullerite-to-diamond transition might proceed more easily in heavy, less stable fullerenes comprising more than 60 carbon atoms in the molecule. We therefore attempted to study explosive compression of fullerenes extracted after separation of the lightest one, $\mathrm{C} 60$.

\section{EXPERIMENTAL PROCEDURE}

Fullerenes were synthesized under low helium pressure in a carbon arc. Then we extracted the fullerenes by toluene from soot that had been subjected to extraction by chloroform. Mass spectroscopy indicates the presence of fullerenes from $\mathrm{C} 60$ to $\mathrm{CI} 00$ (C60 is the main fraction). X-ray structural, TEM and light microscopy investigations indicate three crystal phases in these powders: fcc $\mathrm{C} 60 \mathrm{a}=14.12 \AA$, hcp $\mathrm{C70}$ ( $\mathrm{a}=$ $10.66 \AA, c=17.2 \AA$ ) and a small amount of hcp C60 phase. Cubic $\mathrm{C} 60$ consisted of ellipsoidal particles 1$100 \mu \mathrm{m}$ in size. C70 and hcp fullerite had a more complicated shape, e.g., quadrangles up to $100 \mu \mathrm{m}$ long. Some difficulties may arise when conducting the experiments because of the necessity to provide intense heat transfer from shock-compressed fullerite particles to the surrounding medium in order to retain at ambient conditions the high pressure phases formed on shock-loading. To avoid these difficulties we used the initial powder mixture consisting of fullerite and copper (mass ratio 10/90-2/90), fullerite particles being dispersed in a copper matrix as an efficient quenching medium. The dimensions of the copper particles were in the range of $80-200 \mu \mathrm{m}$.

Bulk-pelleted samples contained in a copper capsule were pressed by a planar shock wave at peak pressures in the range of 24-40 GPa, all pressures exceeding the stability limit (near $20 \mathrm{GPa}$ ) of the fullerene molecule C60 /7,9/. Parameters of these samples are listed in Table 1. All compressed samples were investigated by light microscopy, transmission electron microscopy, $\mathrm{X}$ ray structure and electron probe $\mathrm{X}$-ray microanalysis.

Samples for phase determination were prepared by selective dissolution of copper in $\mathrm{HNO}_{3}$ and separation of carbon phases by centrifugation.

\section{RESULTS AND DISCUSSION}

Diamond, graphite and fcc $\mathrm{C} 60$ were detected in all analyzed specimens (Table 2), while there was no ndiamond or hexagonal diamond (lonsdaleite): only (111) and (220) diffraction peaks were present, belonging to the diamond space group of $\mathrm{Fd} 3 \mathrm{~m}$.

For samples N1, 4, 41 (Table 1), shock-compressed

Table 1

Calculated pressure of explosive compression for copper/fullerite mixture.

\begin{tabular}{|c|c|c|}
\hline $\begin{array}{c}\text { Sample } \\
(\mathrm{N})\end{array}$ & $\begin{array}{c}\text { Initial fullerite } \\
\text { content } w \mathrm{w} \%\end{array}$ & $\begin{array}{c}\text { Peak pressure } \\
\mathrm{GPa}\end{array}$ \\
\hline 1 & 2 & 24 \\
4 & 5 & 24 \\
41 & 10 & 26 \\
3 & 2 & 38 \\
6 & 5 & 38 \\
43 & 10 & 40 \\
\hline
\end{tabular}

Table 2

The indexed X-ray diffraction lines from phases in the sample N6

\begin{tabular}{|l|c|c|c|c|}
\hline $\begin{array}{c}\mathrm{d}_{\mathrm{hkl}} \\
(\AA)\end{array}$ & $\mathrm{I} / \mathrm{I}_{\mathrm{o}}$ & $\begin{array}{c}\text { Diamond } \\
(\mathrm{hkl}) \\
\mathrm{a}=3,55 \AA\end{array}$ & $\begin{array}{c}\text { fcc C60 } \\
(\mathrm{hkl}) \\
\mathrm{a}=14,08 \AA\end{array}$ & $\begin{array}{c}\text { Graphite } \\
(\mathrm{hkl})\end{array}$ \\
\hline 4,96 & $\mathrm{~s}$ & & $(220)$ & \\
4,23 & $\mathrm{~s}$ & & $(311)$ & \\
4,07 & $\mathrm{~m}$ & & $(222)$ & \\
$3,41^{*}$ & $\mathrm{~s}$ & & & $(002)$ \\
3,21 & $\mathrm{~m}$ & & $(331)$ & \\
2,87 & $\mathrm{~m}$ & & $(422)$ & \\
22,71 & $\mathrm{~m}$ & & $(333)$ & \\
& & & $(511)$ & \\
2,05 & vs & $(111)$ & & \\
1,97 & vw & & $(711)$ \\
& & & $(551)$ \\
1,25 & $\mathrm{~s}$ & $(220)$ & \\
\hline
\end{tabular}

*Diffuse line 
at 24-26 GPa, the diamond diffraction line width depends on the content of fullerenes in the initial copper-fullerene mixture. The line width of the samples $\mathrm{Nl}$ and $\mathrm{N} 4 \mathrm{l}$ is larger than that of the sample N4, which means that the average size of diamond crystallites in the sample $\mathrm{N} 4$ is larger than that of the samples $\mathrm{Nl}$ and N41.

At a higher shock loading pressure (up to $38-40$ $\mathrm{GPa}$ ), the phase composition of the samples does not change, except for a slight increase in the diamond content and a slight decrease in the fcc $\mathrm{C} 60$ content according to observed changes of diffraction peak intensities. Narrow diffraction lines of diamond are typical of both the $5 \%$ sample (N6) and the $10 \%$ sample (N43). For the $2 \%$ sample (N3) the diffraction peaks remain diffused as in the case of shock compression at $24 \mathrm{GPa}$.

Fig. 1 presents the X-rayogram of sample N6. It is clearly seen that the diamond diffraction lines consist of separate spots, i.e., the size of the diamond particles reaches $1 \mu \mathrm{m}$. Thus the size of the diamond particles synthesized from fullerenes in our experiments was one order of magnitude larger than that of diamonds synthesized from fullerenes by other investigators $15,10 \%$. TEM investigation of the sample $\mathrm{N} 6$ after separation confirms that the size of the diamond crystallites is nearly $1 \mu \mathrm{m}$ (Fig. 2).

Thus our experimental data allows us to confirm that a considerable increase in shock wave pressure leads to the synthesis of $0.1-1 \mu \mathrm{m}$ size diamonds in a more extended range of initial copper-fullerite powder compositions. Beside diamond, all studied compacts contain considerable amounts of graphite and fcc fullerite, $\mathrm{C} 60$. The presence of $\mathrm{C} 60$ is due to its high stability. Apparently diamonds are mainly synthesized from heavy fullerenes containing $\mathrm{C} 60-\mathrm{C} 100$ in the initial powder.

\section{CONCLUSIONS}

1. Shock-wave synthesis of diamond from fullerene C60-C100-powders was performed using the explosive compaction technique with plane shock-wave loading at different pressures in the range of 24-40 GPa. Compared with methods using a gas gun, this technique is very promising for industrial technologies.

2. The compacts of different initial compositions consisted of diamond, fcc $\mathrm{C} 60$, graphite and amorphous carbon. The coarsest diamond crystallites, $0.1-1 \mu \mathrm{m}$ in diameter, were formed at shock pressures of 24 and 38 $\mathrm{GPa}$ in the compacts of an initial powder mixture copper - 5\% fullerite and at a shock pressure of $40 \mathrm{GPa}$ in the compact of an initial powder mixture copper fullerite.

3. Shock wave synthesis of diamond is performed without the formation of intermediate diamond-like phases, such as $\mathrm{n}$-diamond and lonsdaleite (hexagonal diamond) in the final products.

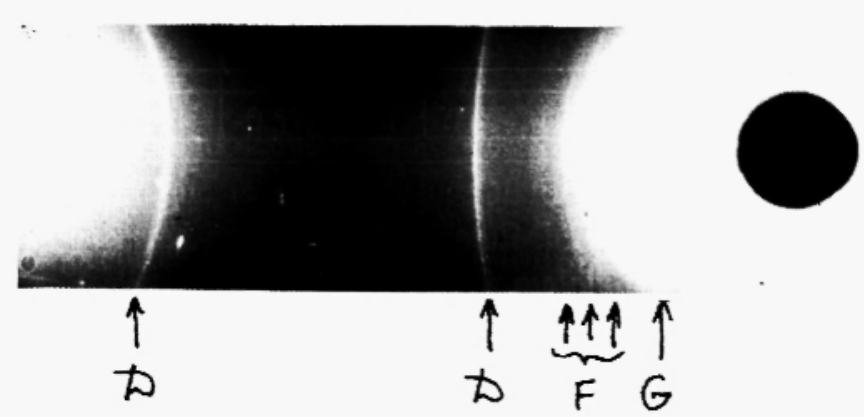

Fig. 1: X-rayogram from sample N6 after shockwave compression. (D - diamond; F - fcc C60; G- graphite)

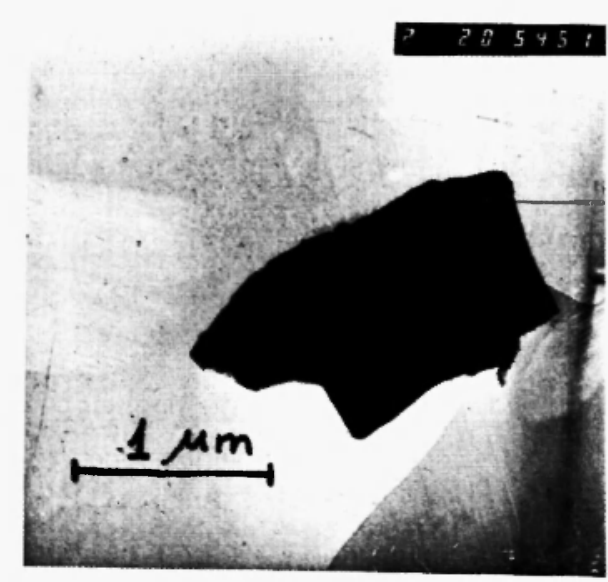

Fig. 2: TEM pattern of diamond separated from sample N6. 


\section{REFERENCES}

1. Kroto, H.W., Heath, J.R., O'Brien, S.C. et al., Nature, 318, 162 (1985).

2. Kratschmer, W., Lamb, L.D., Fostiropulos, K. et al., Nature, 347, 354 (1990).

3. Duclos, S.J., Brister, K., Haddon, R.C. et al., Nature, 351, 380 (1991).

4. Yoo, C.S. and Nellis, W.J., Science, 254, 1489 (1991).

5. Nunez, R.M., Monceau, P. and Hodeau, J.-L., Nature, 355, 237 (1992).
6. Yoo, C.S., Nellis, W.J., Sattler, M.L. et al., Appl. Phys. Lett., 61, 273 (1992).

7. Sekine, T., Proc. Japan. Acad., 68B, 95 (1992).

8. Ruoff, R.S. and Ruoff, A.L., Nature, 350, 663 (1991).

9. Epanchintsev, O.G. and Dityat'ev, A.A., Fisika gorenia i vzryva, N2, 30 (1994).

10. Nellis, W.J. and Yoo, C.S., "Fullerenes subjected to high pressure", in: Conf. of Amer. Electrochem. Soc., Los Angeles, CA, 2-9 June, 1994. 